\title{
Sedimentation of Concentrated Spherical Particles with a Charge-Regulated Surface
}

\author{
Eric Lee, Tsai-Shih Tong, Ming-Hui Chih, and Jyh-Ping Hsu ${ }^{1}$ \\ Department of Chemical Engineering, National Taiwan University, Taipei, Taiwan 10617, Republic of China
}

Received January 14, 2002; accepted March 25, 2002; published online June 3, 2002

The sedimentation of a concentrated colloidal dispersion is examined for the case of an arbitrary double-layer thickness. Here, a general mixed-type condition on particle surface is assumed, and the classic models, which assume constant surface properties, can be recovered as the special cases of the present analysis. In particular, the behavior of biological cells, which carry dissociable functional groups on their surfaces, and particles, which are capable of exchanging ions with the surrounding medium, can be simulated by the present model. The mixed-type boundary condition leads to several interesting results in both sedimentation velocity and sedimentation potential as double-layer thickness and the concentration of particles vary. (c) 2002 Elsevier Science (USA)

Key Words: sedimentation; concentrated spherical dispersion; charge-regulated surface.

\section{INTRODUCTION}

Gravitational sedimentation of charged colloidal particles is a classic problem, which, together with electrophoresis and electroosmotic flow, consists of the so-called electrokinetic phenomena. Here, the charged condition on a particle surface plays a significant role in the determination of its behavior. Von Smoluchowski (1), for example, concluded that if the electrical double layer surrounding a particle is infinitely thin, then its sedimentation velocity is slower than that of an uncharged particle by a term proportional to the square of surface potential. The problem involves an arbitrary thick double layer discussed by Booth (2, 3), Overbeek (4), and Saville (5). The sedimentation of charged particles in an infinite medium was investigated by Ohshima et al. (6). They showed that an approximate analytical expression for both the sedimentation potential and the sedimentation velocity can be derived if the surface potential is low and the double layer is thin. A numerical scheme is necessary, however, for the case of arbitrary surface potential and double-layer thickness. Keh and Ding (7) considered the sedimentation of a concentrated dispersion of spherical particles under the condition of low surface potential. To examine the ef-

\footnotetext{
${ }^{1}$ To whom correspondence should be addressed. Fax: 886-2-23623040. E-mail: jphsu@ccms.ntu.edu.tw.
}

fect of the presence of a boundary on sedimentation, Pujar and Zydney (8) considered a charged particle in a spherical cavity under the conditions of small Peclet number and low surface potential. The cavity was found to have the effect of increasing the magnitude of the excess force if the double layer surrounding the particle is thin, but the reverse is true if the double layer is thick.

Previous analyses are almost always based on either constant surface potential or constant surface charge. These conditions, although making the mathematical treatment simpler, represent only limiting or idealized cases, which can be unrealistic in practice (9). In the case of biocolloids or particles covered by an artificial membrane, for example, the charged conditions on the particle surface are governed by the degree of dissociation of the functional groups as a response to the variation in the nearby environment (9-16). Here, the constant surface potential and the constant surface charge models correspond, respectively, to the cases when the dissociation reactions of the functional groups are infinitely fast and infinitely slow. Ding and Keh (17) analyzed the problem of concentrated spherical dispersion for the case of arbitrary double-layer thickness. Although a charge-regulation condition is adopted, it is considered as a perturbed term rather than as an actual condition on the particle surface.

In the present study, the sedimentation of monodispersed spherical colloids is discussed. The surface charge of a particle arises from the dissociation of the functional groups on its surface. This leads to a mixed-type boundary condition on the particle surface, which is a generalization of the conventional constant surface potential and constant surface-charged density models (18). Furthermore, both the concentration of the dispersed phase and the thickness of the double layer surrounding a particle can be arbitrary. The latter implies that the interaction between neighboring double layers needs to be taken into account.

\section{THEORY}

Let us consider monodispersed spherical particles of radius $a$ in a $z_{1}: z_{2}$ electrolyte solution, $z_{1}$ and $z_{2}$ being the valences of cations and anions, respectively. The concentration of the dispersed phase may be appreciable so that the interaction 


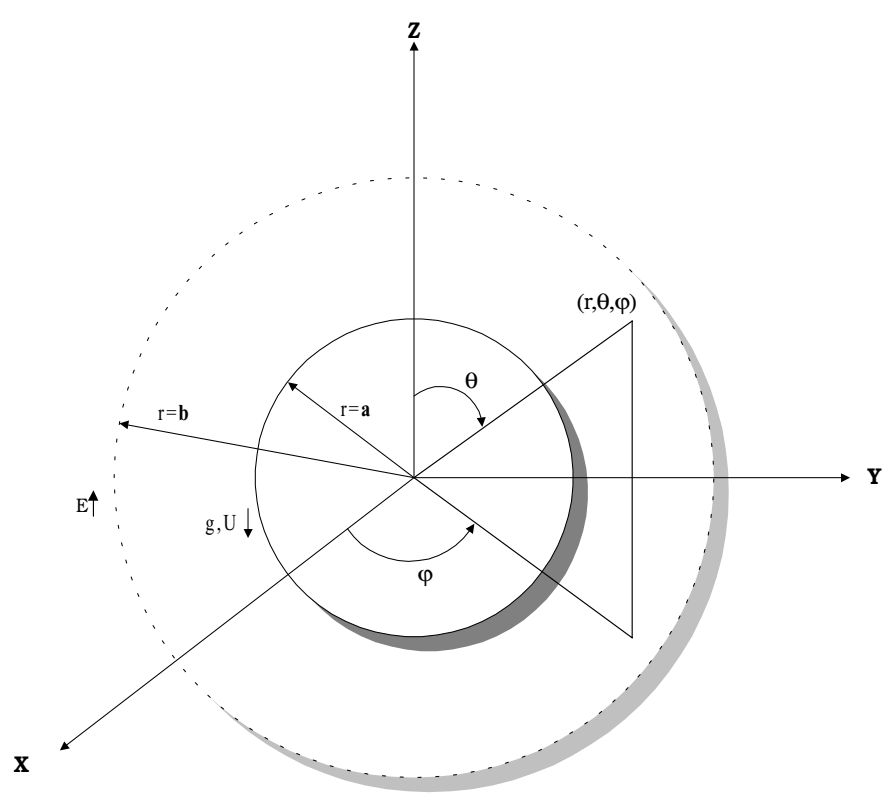

FIG. 1. Schematic representation of the cell model. A representative particle of radius $a$ is enclosed by a concentric liquid spherical shell of radius $b$. $\mathbf{U}$ is the sedimentation velocity of the particle, $\mathbf{g}$ is gravity, and $\mathbf{E}$ is the induced electric field.

between adjacent particles needs to be considered. Suppose that the surface of a particle contains dissociable functional group $\mathrm{AH}$, which is capable of undergoing the dissociation reaction

$$
\mathrm{AH} \Leftrightarrow \mathrm{A}^{-}+\mathrm{H}^{+}
$$

Note that this yields a negatively charged surface. Let $N_{\mathrm{s}}$ be the concentration of AH. As illustrated in Fig. 1, the cell model of Kuwabara (19), where the system under consideration comprises spherical particles each enclosed by a concentric spherical liquid shell of radius $b$, is applied. The volume fraction of the dispersed phase can be measured by the ratio $\lambda=(a / b)^{3}$. Due to the influence of gravity $\mathbf{g}$, the particles in the dispersion move in the $-z$ direction, and an electric field $\mathbf{E}$ that is in the $z$ direction is induced. The spherical coordinates $(r, \theta, \varphi)$ with the origin located at the center of a representative particle are adopted.

At steady state, the conservation of ionic species $j$ in the liquid phase leads to

$$
\nabla \bullet\left[D_{j}\left[\nabla n_{j}+\frac{z_{j} e n_{j}}{k_{\mathrm{B}} T} \nabla \phi\right]-n_{j} \mathbf{v}\right]=0, \quad j=1,2,
$$

where $\nabla$ is the gradient operator; $D_{j}, n_{j}$, and $z_{j}$ are respectively the diffusivity, the number concentration, and the valence of ionic species $j ; e, k_{\mathrm{B}}$, and $T$ denote respectively the elementary charge, the Boltzmann constant, and the absolute temperature; and $\phi$ and $\mathbf{v}$ are respectively the electrical potential and the velocity. If we let $n_{10}$ and $n_{20}$ be the equilibrium concentrations of cations and anions respectively, then the electroneutrality in the bulk liquid phase requires that $n_{20}=\left(n_{10} / \alpha\right)$ with $\alpha=-z_{2} / z_{1}$.
Suppose that the spatial variation of the electrical potential at equilibrium can be described by the Poisson-Boltzmann equation

$$
\nabla^{2} \phi=-\frac{\rho}{\varepsilon}=-\sum_{j=1}^{2} \frac{z_{j} e n_{j}}{\varepsilon},
$$

where $\varepsilon$ is the permittivty of the liquid phase, and $\rho$ is the space charge density.

In the creeping flow regime the flow field can be described by

$$
\begin{aligned}
\nabla \bullet \mathbf{v} & =0 \\
\eta \nabla^{2} \mathbf{v}-\nabla p-\rho \nabla \phi & =0,
\end{aligned}
$$

where $p$ and $\eta$ denote respectively the pressure and the viscosity of liquid phase. Here we assume that the latter is incompressible and has constant physical properties.

Following the treatment of O'Brien and White (20), the electrical potential $\phi$ is decomposed into two terms, namely, the electrical potential that would exist in the absence of the induced electric field (or the equilibrium electrical potential), $\phi_{1}$, and the electrical potential arising from the induced electric field, $\phi_{2}$; that is, $\phi=\phi_{1}+\phi_{2}$. Suppose that the spatial distribution of ionic species $j$ can be described by (20)

$$
n_{j}=n_{j 0} \exp \left(-\frac{z_{j} e\left(\phi_{1}+\phi_{2}+g_{j}\right)}{k_{\mathrm{B}} T}\right), \quad j=1,2,
$$

where the perturbed function $g_{j}$ simulates the effect of flow field on the spatial distribution of ion concentration. If the induced electric field is weak, then both $\phi_{2}$ and $g_{j}$ are small compared to $\phi_{1}$ (20). In this case Eq. [6] can be approximated by

$$
n_{j}^{*}=\exp \left(-z_{j} \phi_{1}^{*}\right)\left[1-z_{j}\left(\phi_{2}^{*}+g_{j}^{*}\right)\right], \quad j=1,2,
$$

where $n_{j}^{*}=n_{j} / n_{j 0}, \phi_{j}^{*}=\phi_{j} /\left(k_{\mathrm{B}} T / e\right)$, and $g_{j}^{*}=g_{j} /\left(k_{\mathrm{B}} T / e\right)$.

For a simpler mathematical treatment the governing equations are rewritten in scaled forms. Here, particle radius $a$ is chosen as the scale factor for length, the velocity of a particle predicted by the Smoluchowski theory when an electric field of strength $\left[\left(k_{\mathrm{B}} T / e\right) / a\right]$ is applied, $U_{\mathrm{E}}=\left[\varepsilon\left(k_{\mathrm{B}} T / e\right)^{2} / \eta a\right]$ is chosen as the scaled factor for velocity, and $\left(k_{\mathrm{B}} T / e\right)$ is adopted as the scaled factor for electrical potential. It can be shown (21) that the following equations need to be solved simultaneously (see also the Appendix):

$$
\begin{gathered}
\nabla^{2} \phi_{1}^{*}=-\frac{(\kappa a)^{2}}{(1+\alpha)}\left[\exp \left(-\phi_{1}^{*}\right)-\exp \left(\alpha \phi_{1}^{*}\right)\right] \\
{\left[L-\frac{(\kappa a)^{2}}{1+\alpha}\left[\exp \left(-\phi_{1}^{*}\right)+\alpha \exp \left(\alpha \phi_{1}^{*}\right)\right]\right] \Phi_{2}} \\
=\frac{(\kappa a)^{2}}{1+\alpha}\left[G_{1} \exp \left(-\phi_{1}^{*}\right)+\alpha G_{2} \exp \left(\alpha \phi_{1}^{*}\right)\right]
\end{gathered}
$$




$$
\begin{gathered}
L G_{1}-\frac{d \phi_{1}^{*}}{d r^{*}} \frac{d G_{1}}{d r^{*}}=\operatorname{Pe}_{1} v_{r}^{*} \frac{d \phi_{1}^{*}}{d r^{*}} \\
L G_{2}+\alpha \frac{d \phi_{1}^{*}}{d r^{*}} \frac{d G_{2}}{d r^{*}}=\operatorname{Pe}_{2} v_{r}^{*} \frac{d \phi_{1}^{*}}{d r^{*}}
\end{gathered}
$$

$D^{4} \Psi=-\frac{(\kappa a)^{2}}{1+\alpha}\left[\left(\exp \left(-\phi_{1}^{*}\right) G_{1}+\alpha \exp \left(\alpha \phi_{1}^{*}\right) G_{2}\right) \frac{d \phi_{1}^{*}}{d r^{*}}\right]$.

In these expressions $\mathrm{Pe}_{j}=\varepsilon\left(z_{j} e / k_{\mathrm{B}} T\right)^{2} / \eta D_{j}, j=1,2$, is the electric Peclet number for ion species $j$, and $G_{1}(r)$ and $G_{2}(r)$ are respectively the radial part of $g_{1}^{*}$ and that of $g_{2}^{*}$. The reciprocal Debye length, $\kappa$, and the operators $L$ and $D^{4}$ are defined by

$$
\begin{aligned}
\kappa & =\left[\sum_{j=1}^{2} n_{j 0}\left(e z_{j}\right)^{2} / \varepsilon k_{\mathrm{B}} T\right]^{1 / 2} \\
L & \equiv \frac{d^{2}}{d r^{* 2}}+\frac{2}{r^{*}} \frac{d}{d r^{*}}-\frac{2}{r^{* 2}} \\
D^{4} & =\left(D^{2}\right)^{2}=\left(\frac{d^{2}}{d r^{* 2}}-\frac{2}{r^{* 2}}\right)^{2} .
\end{aligned}
$$

The boundary conditions associated with Eqs. [8]-[12] are

$$
\begin{aligned}
& \frac{d \phi_{1}^{*}}{d r^{*}}=\frac{A}{1+B \exp \left(-\phi_{1}^{*}\right)}, \quad r^{*}=1 \\
& \frac{d \phi_{1}^{*}}{d r^{*}}=0, \quad r^{*}=b / a \\
& \frac{d \Phi_{2}}{d r^{*}}=0, \quad r^{*}=1 \\
& \frac{d \Phi_{2}}{d r^{*}}=-E_{z}^{*}, \quad r^{*}=b / a \\
& \frac{d G_{j}}{d r^{*}}=0, \quad r^{*}=1, \quad j=1,2 \\
& G_{j}=-\Phi_{2}, \quad r^{*}=b / a, \quad j=1,2 \\
& \Psi=-\frac{1}{2} U^{*} r^{* 2} \quad \text { and } \quad \frac{d \Psi}{d r^{*}}=-U^{*} r^{*}, \quad r^{*}=1 \\
& \Psi=0 \quad \text { and } \quad \Psi=\left[\frac{1}{r^{*}} \frac{d^{2} \Psi}{d r^{* 2}}-\frac{2}{r^{* 3}}\right] \Psi=0, \quad r^{*}=b / a .
\end{aligned}
$$

In these expressions, $E_{z}^{*}=E_{z} a /\left(k_{\mathrm{B}} T / e\right)$, and $U^{*}=U / U_{E}, E_{z}$ and $U$ being respectively the $z$ component of the electrical field and the terminal velocity of a particle. The origin of Eq. [16] is elaborated in the Appendix. Equation [17] implies that the unit cell as a whole is electrically neutral. Equation [18] is a Neumann-type condition (22), which implies that the particle is nonconductive, and its surface is impermeable to ions. Equation [19] denotes the applied electric field. Equation [20] suggests the surface of a particle is impenetrable to liquid phase. Equation [21] implies that there is no net ionic flux across the virtual surface, which leads to $g_{i}+\phi_{2}=0$. Equations [22] and
[23] describe the condition of the flow field. At steady state, the magnitude of the velocity of a particle being $U$ at the virtual surface $(r=b)$ remains constant and satisfies Kuwabara's model of zero vorticity.

The scaled sedimentation potential, $E^{*} / U^{*}$, can be evaluated based on the fact that the sedimentation of particles generates no net current. Employing this condition on the horizontal plane $\theta=\pi / 2$ yields $(23)$

$$
\begin{aligned}
\langle\mathbf{i}\rangle & =0=\left.2 \pi \int_{a}^{b} \mathbf{r} \cdot i_{\theta} d r\right|_{\theta=\pi / 2} \\
& =\left.2 \pi \int_{a}^{b} \mathbf{r} \cdot\left(\sum_{j=1}^{2} z_{j} e n_{j} v_{j \theta}\right) d r\right|_{\theta=\pi / 2},
\end{aligned}
$$

where $i_{\theta}$ and $v_{j \theta}$ are the $\theta$ component of the electric current $\mathbf{i}$ and that of the flow velocity of the $j$ th ionic species, $\mathbf{v}_{j}$, defined respectively as

$$
\mathbf{i}=\sum_{j=1}^{2} z_{j} e n_{j} \mathbf{v}_{j}
$$

and

$$
\mathbf{v}_{j}=\mathbf{v}-D_{j}\left(\frac{z_{j} e}{k_{\mathrm{B}} T} \nabla \phi+\frac{\nabla n_{j}}{n_{j}}\right) .
$$

It can be shown that (23)

$$
\begin{aligned}
\mathbf{i}= & \frac{\varepsilon^{2}}{\eta \mathrm{a}^{3}}\left(\frac{k_{\mathrm{B}} T}{e}\right)^{3} \frac{(\kappa a)^{2}}{(1+\alpha)}\left\{\left[\exp \left(-\phi_{1}^{*}\right)+\exp \left(\alpha \phi_{1}^{*}\right)\right] v^{*}\right. \\
& \left.+\left[\frac{1}{\mathrm{Pe}_{1}} \exp \left(-\phi_{1}^{*}\right) \nabla^{*} g_{1}^{*}+\frac{\alpha}{\mathrm{Pe}_{2}} \exp \left(\alpha \phi_{1}^{*}\right) \nabla^{*} g_{2}^{*}\right]\right\} \\
i_{\theta}= & \frac{\varepsilon^{2}}{\eta \mathrm{a}^{3}}\left(\frac{k_{\mathrm{B}} T}{e}\right)^{3} \frac{(\kappa a)^{2}}{(1+\alpha)}\left\{\left[\exp \left(-\phi_{1}^{*}\right)+\exp \left(\alpha \phi_{1}^{*}\right)\right] \frac{d \Psi}{d r^{*}}\right. \\
& \left.-\left[\frac{1}{\mathrm{Pe}_{1}} \exp \left(-\phi_{1}^{*}\right) G_{1}^{*}+\frac{\alpha}{\mathrm{Pe}_{2}} \exp \left(\alpha \phi_{1}^{*}\right) G_{1}^{*}\right]\right\} \frac{\sin \theta}{r^{*}} .
\end{aligned}
$$

This expression can be rewritten as

$$
i_{\theta}=I_{\mathrm{a}} I_{\theta}\left(r^{*}\right) \frac{\sin \theta}{r^{*}},
$$

where

$$
\begin{aligned}
I_{\mathrm{a}}= & \frac{\varepsilon^{2}}{\eta a^{3}}\left(\frac{k_{\mathrm{B}} T}{z_{1} e}\right)^{3} \frac{(\kappa a)^{2}}{(1+\alpha)}, \\
I_{\theta}\left(r^{*}\right)= & \left\{\left[\exp \left(-\phi_{1}^{*}\right)+\exp \left(\alpha \phi_{1}^{*}\right)\right] \frac{d \Psi}{d r^{*}}\right. \\
& \left.-\left[\frac{1}{\mathrm{Pe}_{1}} \exp \left(-\phi_{1}^{*}\right) G_{1}+\alpha \frac{1}{\mathrm{Pe}_{2}} \exp \left(\alpha \phi_{1}^{*}\right) G_{2}\right]\right\} .
\end{aligned}
$$


Substituting Eq. [28] into Eq. [24], we obtain

$$
\langle\mathbf{i}\rangle=0=2 \pi a^{2} I_{\mathrm{a}} \int_{1}^{b / a} I_{\theta}\left(r^{*}\right) d r^{*} .
$$

Equation [31] suggests that $I_{\theta}\left(r^{*}\right)$ comprises the current due to the convective motion of the liquid phase, $I_{\theta, \mathrm{c}}$, and that due to the diffusion of ionic species, $I_{\theta, \mathrm{d}}$, where

$$
\begin{aligned}
I_{\theta, \mathrm{c}} & =\left[\exp \left(-\phi_{1}^{*}\right)-\exp \left(\alpha \phi_{1}^{*}\right)\right] \frac{d \Psi}{d r^{*}} \\
I_{\theta, \mathrm{d}} & =-\left[\frac{1}{\mathrm{Pe}_{1}} \exp \left(-\phi_{1}^{*}\right) G_{1}+\frac{\alpha}{\mathrm{Pe}_{2}} \exp \left(\alpha \phi_{1}^{*}\right) G_{2}\right] .
\end{aligned}
$$

The phenomenon under consideration can be decomposed into two problems $(20,21)$. In the first problem a representative particle moves at a constant velocity in the absence of the induced electric field. In the second problem the representative particle remains fixed in the prescene of the induced electric field. It can be shown that $\langle i\rangle_{1}=\delta U^{*}$ and $\langle i\rangle_{2}=\beta E^{*}$, where $\langle i\rangle_{1}$ and $\langle i\rangle_{2}$ are the net currents across the plane $\theta=\pi / 2$ in problems 1 and 2 , respectively. Since the net current vanishes on $\theta=\pi / 2$, we have $E^{*} / U^{*}=-\delta / \beta$. Let $F_{E z}$ and $F_{D z}$ be the electric force and the hydrodynamic forces acting on the representative particle, and $F_{g}$ be the gravitational force. Then (23)

$$
\begin{aligned}
F_{E z}= & \frac{8}{3} \pi \varepsilon\left(\frac{k_{\mathrm{B}} T}{e}\right)^{2}\left(r^{*} \frac{d \phi_{1}^{*}}{d r^{*}} \Phi_{2}\right)_{r^{*}=1} \\
= & \frac{8}{3} \pi \varepsilon\left(\frac{k_{\mathrm{B}} T}{e}\right)^{2} F_{E z}^{*} \\
F_{D z}= & \frac{4}{3} \pi \varepsilon\left(\frac{k_{\mathrm{B}} T}{e}\right)^{2}\left(r^{* 4} \frac{\partial}{\partial r^{*}}\left(\frac{D^{2} \Psi}{r^{* 2}}\right)\right)_{r^{*}=1}+\frac{4}{3} \pi \varepsilon\left(\frac{k_{\mathrm{B}} T}{e}\right)^{2} \\
& \times \frac{(\kappa a)^{2}}{(1+\alpha)}\left[r^{* 2}\left[\exp \left(-\phi_{1}^{*}\right)-\exp \left(\alpha \phi_{1}^{*}\right)\right] \Phi_{2}\right]_{r^{*}=1} \\
= & \frac{4}{3} \pi \varepsilon\left(\frac{k_{\mathrm{B}} T}{e}\right)^{2}\left(F_{D h z}^{*}+F_{D e z}^{*}\right) \\
F_{g}= & \frac{4}{3} \pi a^{3}\left(\rho_{p}-\rho_{f}\right) g .
\end{aligned}
$$

\section{Effect of $A$}

Figure 2 illustrates the variation of the scaled surface potential $\phi_{1 \mathrm{~S}}^{*}$ as a function of $\kappa a$ at various $A\left(=e^{2} N_{\mathrm{s}} a / \varepsilon k_{\mathrm{B}} T\right)$. This figure reveals that for a fixed $\kappa a$, the larger the value of $A$, the higher the value of $\left|\phi_{1 S}^{*}\right|$. This is because the larger the value of $A$, the higher the concentration of the dissociable functional groups on the particle surface $N_{\mathrm{s}}$, and, therefore, the higher the surface charge density. Figure 2 also suggests that for a fixed $A,\left|\phi_{1 \mathrm{~S}}^{*}\right|$ decreases with the increase in $\kappa a$. This is because the larger the value of $\kappa a$, the thinner the double layer, which implies the less the available space for mobile ions. This leads to a less degree of dissociation of functional groups.

Figure 3 shows the variation of the scaled sedimentation velocity, $U / U_{0}$, as a function of $\kappa a$ at various $A$. The corresponding variation in the force ratio $f_{2} / f_{1}, f_{1}$ and $f_{2}$ being respectively the total forces in problems 1 and 2, is illustrated in Fig. 4, and that in the scaled sedimentation potential $E^{*} / U^{*}$ is presented in Fig. 5. It is interesting to note that $U / U_{0}$ exhibits both a local minimum and a local maximum as $\kappa a$ varies. This is due to the complicated behavior of the electric and hydrodynamic forces exerted on a particle and that of the sedimentation potential. 


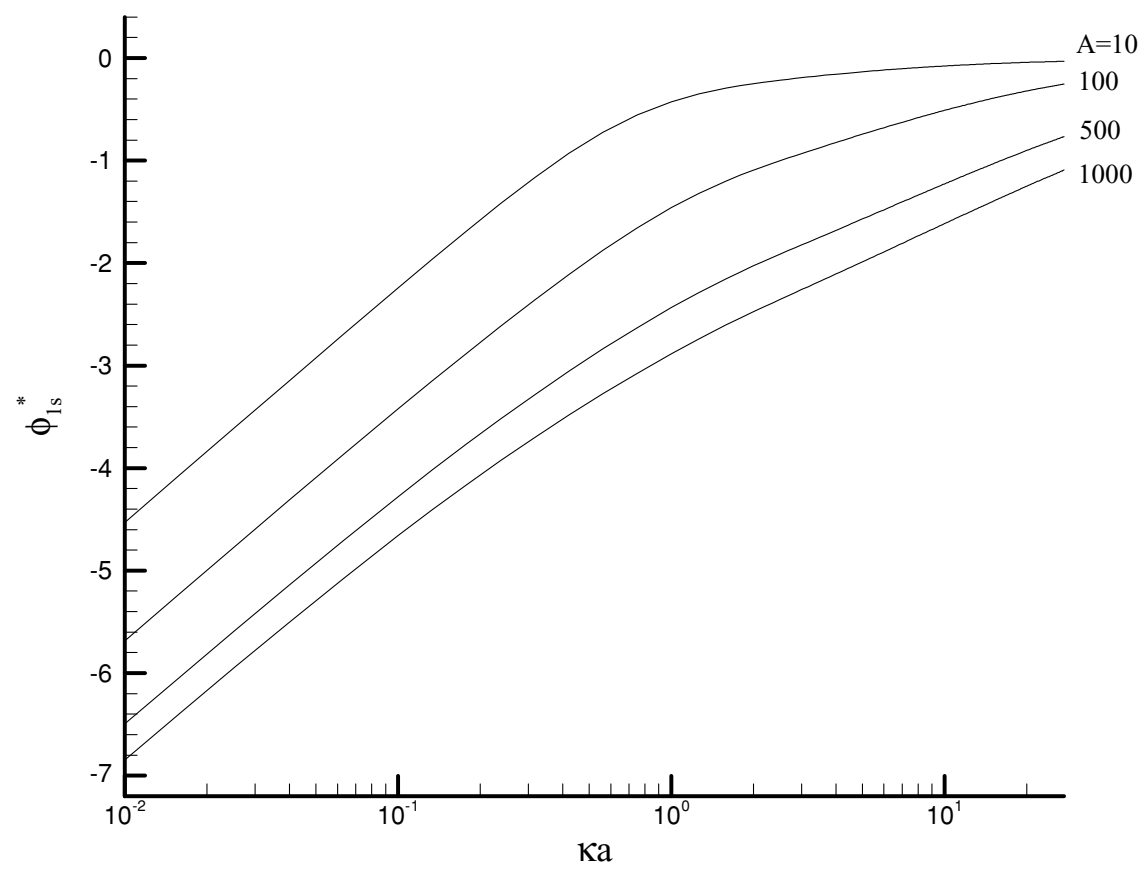

FIG. 2. Variation of scaled surface potential $\phi_{1 \mathrm{~S}}^{*}$ as a function of $\kappa a$ at various $A$ for the case of $B=10 . \operatorname{Key} \lambda=(0.5)^{3}$, and $\mathrm{Pe} e_{1}=\mathrm{Pe}_{2}=0.01$.

$U / U_{0}$ can be expressed as

$$
\frac{U}{U_{0}}=\frac{9}{2}\left[f_{1}\left(1+\frac{f_{2}}{f_{1}} \frac{E^{*}}{U^{*}}\right)\right]^{-1} .
$$

Both the electric force and the hydrodynamic force experienced by a particle depend on both its surface potential and the thickness of the double layer. The presence of the double layer has the effect of retarding the movement of the particle. If $\kappa a$ is small,

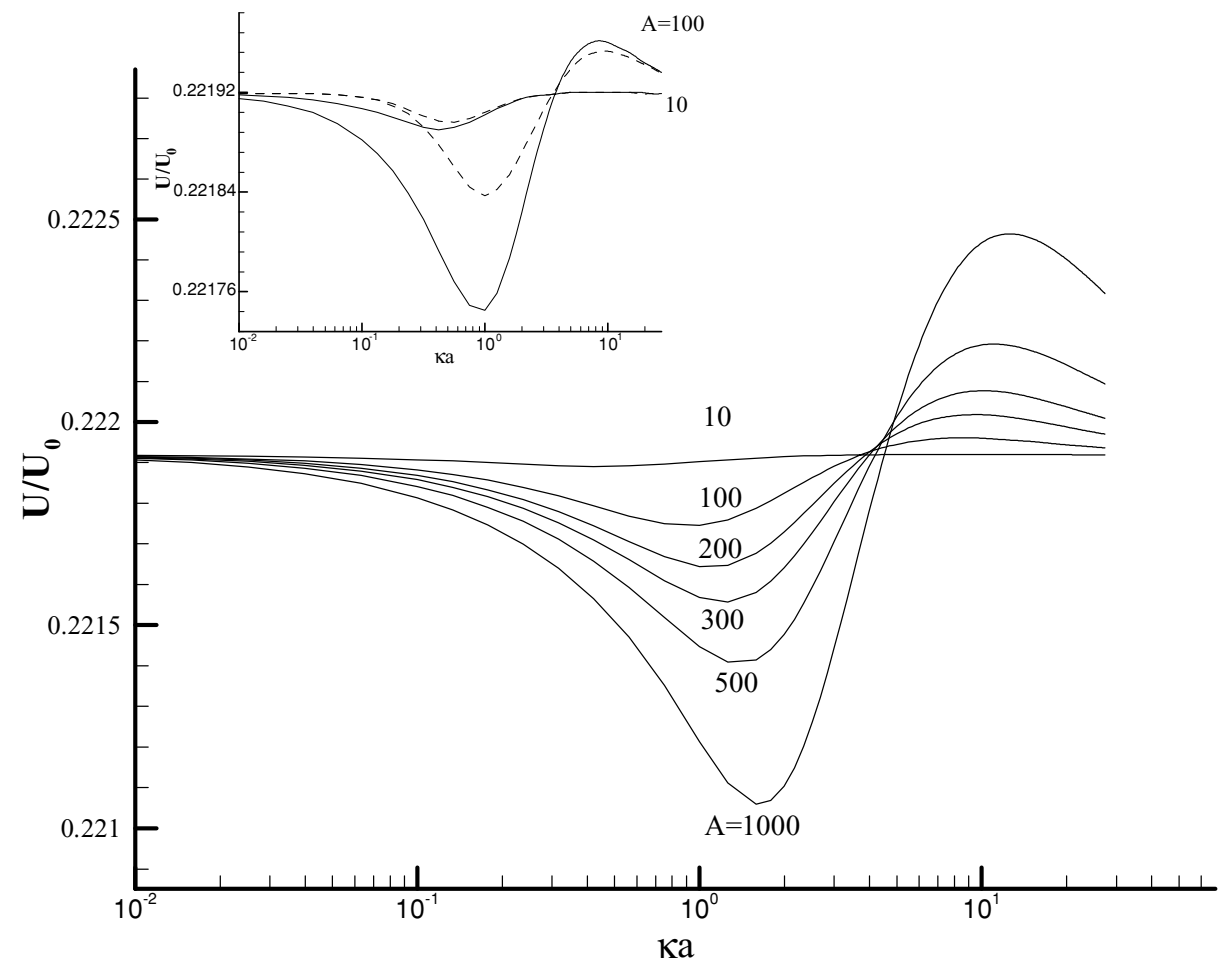

FIG. 3. Variation of scaled sedimentation velocity $U / U_{0}$ as a function of $\kappa a$ at various $A$ for the case of Fig. 2. Dashed lines are results based on the linearized Poisson-Boltzmann equation. 


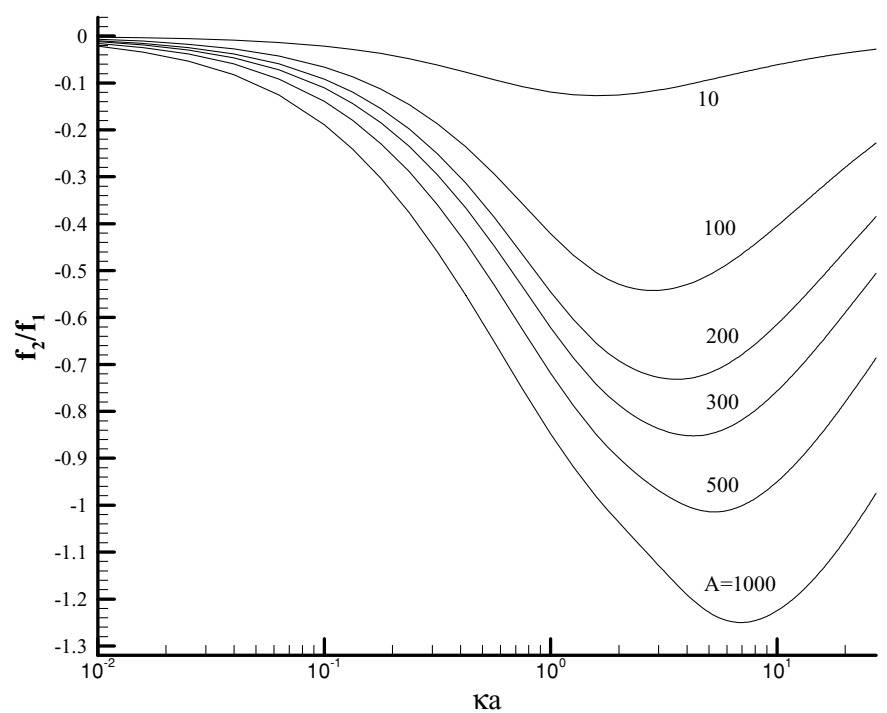

FIG. 4. Variation of force ratio $f_{2} / f_{1}$ as a function of $\kappa a$ for the case of Fig. 2.

the double layer is thick, and the interaction between adjacent double layers leads to a large $f_{1}$. On the other hand, if $\kappa a$ is large, the double layer is thin, the absolute rate of variation in electrical potential near particle surface is large, which leads to a large $f_{2}$. However, as suggested by Fig. 2, a large $\kappa a$ leads to a low $\left|\phi_{1 \mathrm{~S}}^{*}\right|$, which yields a low degree of dissociation of functional groups, and therefore, a slow sedimentation velocity. Figure 3 also suggests that $U / U_{0}$ approaches a constant value for both

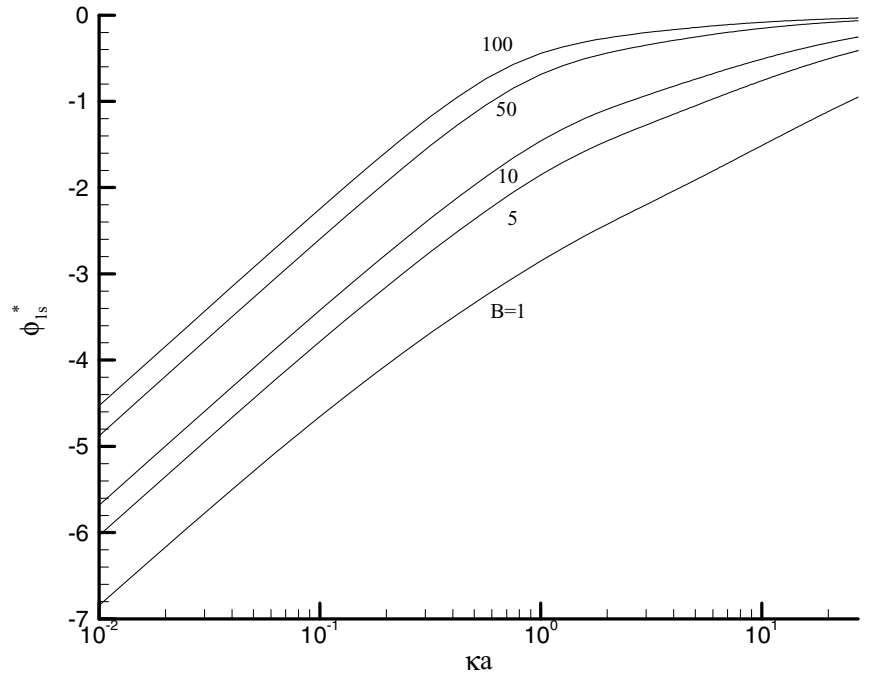

FIG. 6. Variation of scaled surface potential $\phi_{1 \mathrm{~S}}^{*}$ as a function of $\kappa a$ at various $B$ for the case $A=100$. Key: $\lambda=(0.5)^{3}$, and $\mathrm{Pe}_{1}=\mathrm{Pe}_{2}=0.01$. $\kappa a \rightarrow 0$ and $\kappa a \rightarrow \infty$; the limiting values are independent of $A$. The former can be explained by the result shown in Fig. 4. As $\kappa a \rightarrow 0$, the double layer surrounding a particle becomes infinitely thick, and the overlapping between adjacent double layers makes the movement of a particle difficult. In this case, the hydrodynamic drag force, which has the effect of reducing the sedimentation velocity, dominates. Since $A$ is mainly related to electric potential (and therefore, electric force), its magnitude

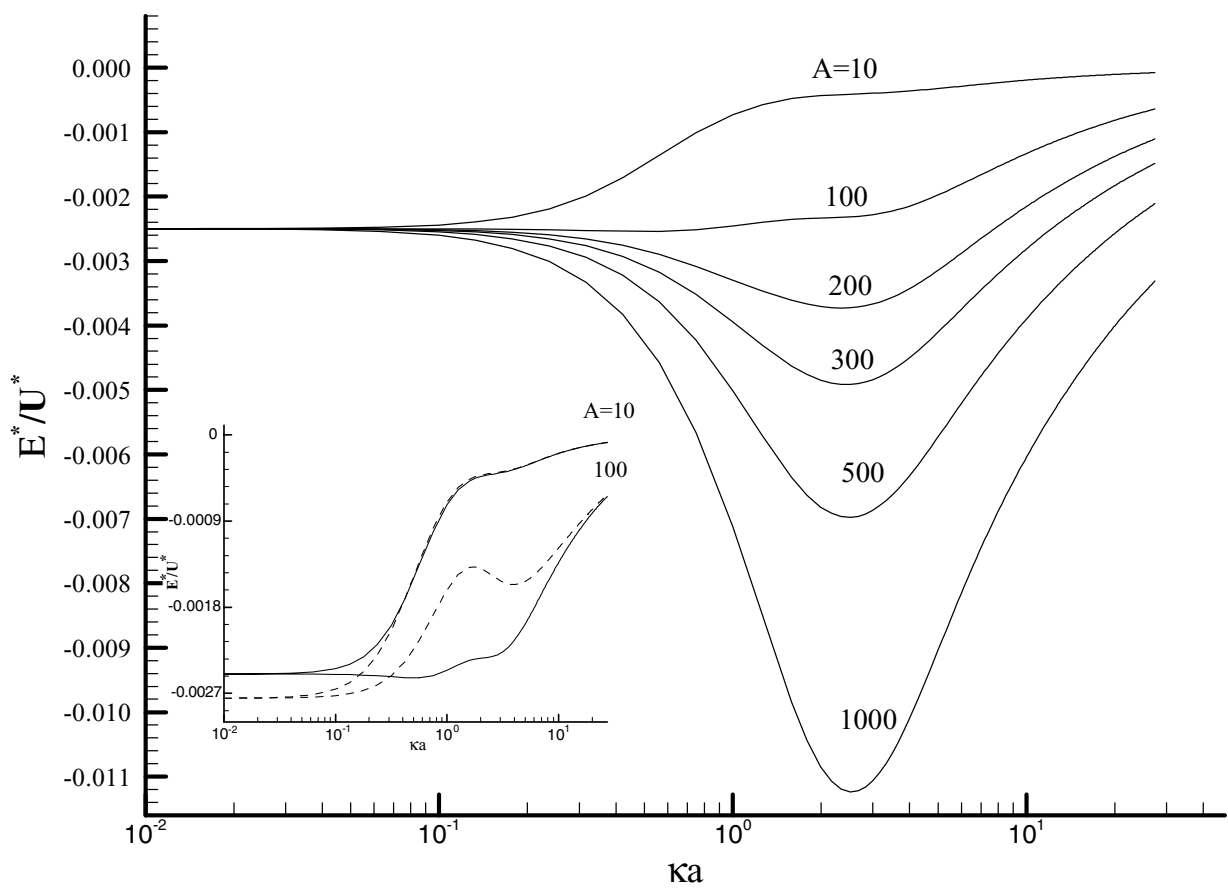

FIG. 5. Variation of scaled sedimentation potential $E^{*} / U^{*}$ as a function of $\kappa a$ at various $A$ for the case $B=10$. Dashed lines are results based on the linearized Poisson-Boltzmann equation. Key: same as that described in the legend to Fig. 2. 


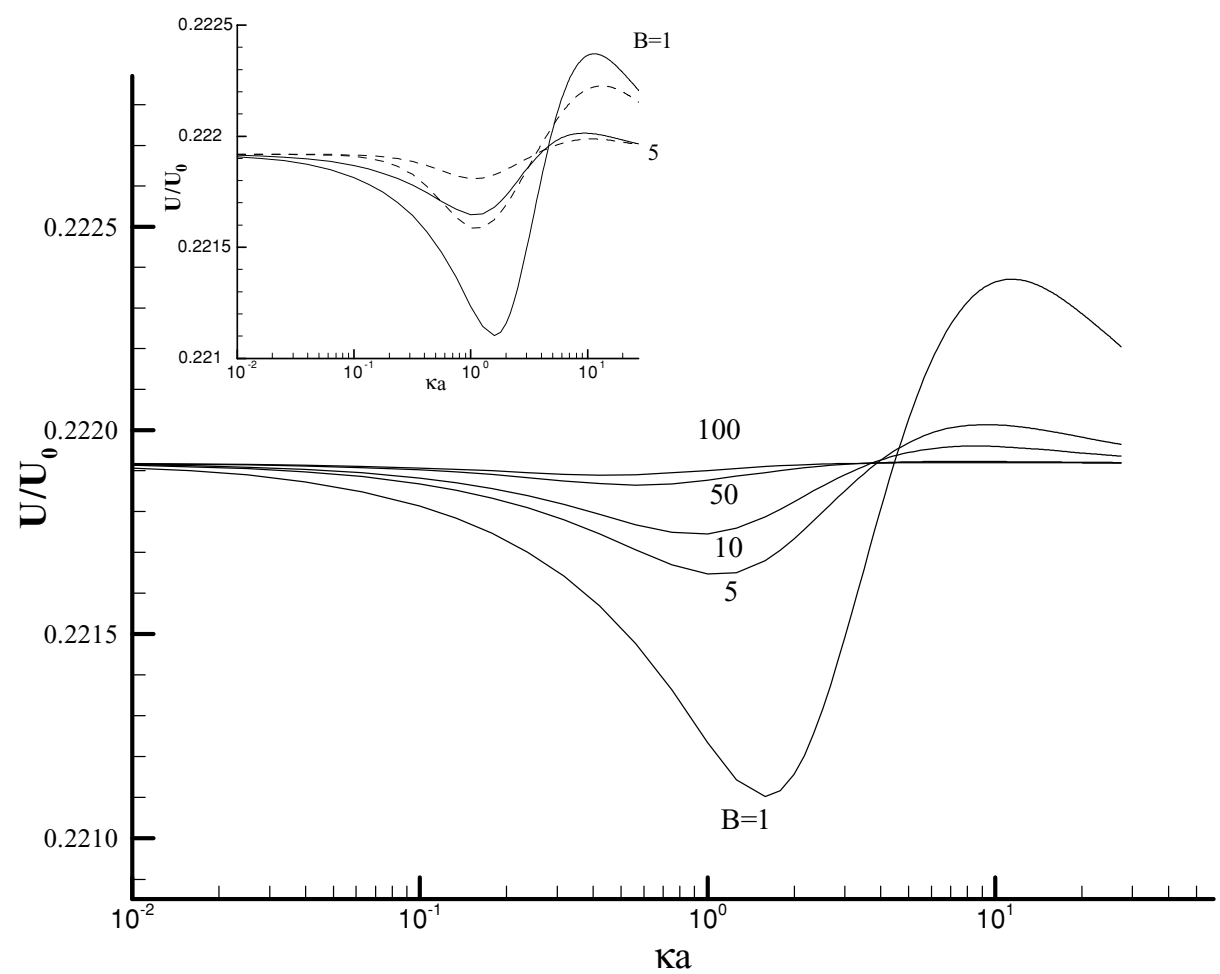

FIG. 7. Variation of scaled sedimentation velocity $U / U_{0}$ as a function of $\kappa a$ at various $B$ for the case $A=100$. Dashed lines are results based on the linearized Poisson-Boltzmann equation. Key: same as that described in the legend to Fig. 2.

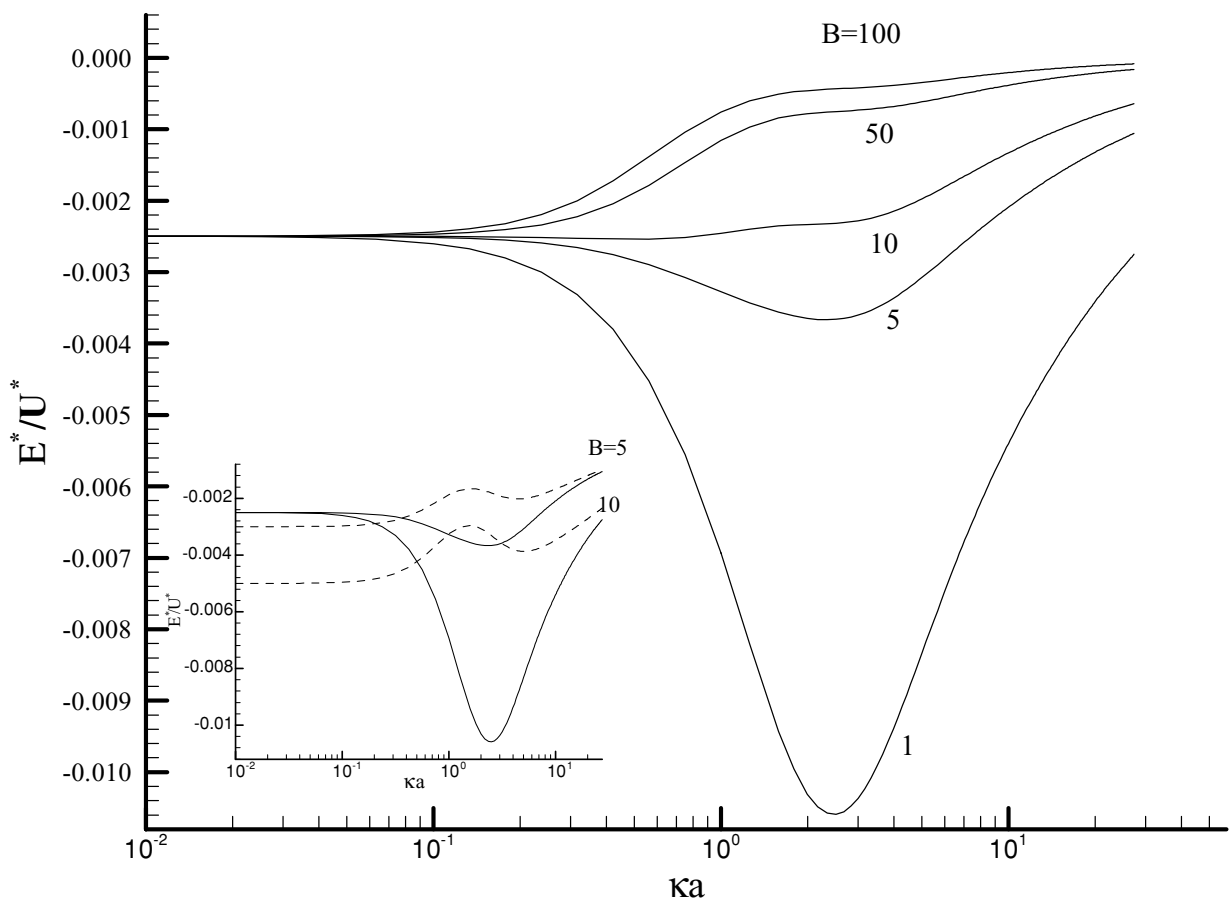

FIG. 8. Variation of scaled sedimentation potential $E^{*} / U^{*}$ and as a function of $\lambda$ for the case of Fig. 6 . Dashed lines are results based on the linearized Poisson-Boltzmann equation. 
becomes insignificant. The latter arises from the fact that the gradient of electrical potential becomes negligible as the double layer becomes infinitely thin. Figure 5 reveals that if $\kappa a \rightarrow 0$, $E^{*} / U^{*}$ approaches a constant value, which is independent of $A$. This is because if $\kappa a \rightarrow 0$, the double layer is infinitely thick, and in this case the current is mainly contributed by the convective motion of ions (21), which is not directly related to $A$. Figure 5 suggests that for a fixed $\kappa a,\left|E^{*} / U^{*}\right|$ increases with the increase in $A$. Since the larger the value of $A$, the higher the $\left|\phi_{1 \mathrm{~S}}^{*}\right|$, as discussed for Fig. 2, this is consistent with the result of Lee et al. (21), in which the sedimentation potential of a particle is found to be linearly dependent on its surface potential. Figure 5 indicates that if $A$ is small, $\left|E^{*} / U^{*}\right|$ decreases monotonically with the increase in $\kappa a$. However, if $A$ is sufficiently large, $\left|E^{*} / U^{*}\right|$ may have a local maximum as $\kappa a$ varies. The calculation of $\left|E^{*} / U^{*}\right|$ is based on the fact that the net current passing the plane $\theta=\pi / 2$ vanishes. According to Eq. [27], the current comprises two elements, namely, the convective motion and the diffusive motion of ions. As pointed out by Lee et al. (21), for the case of low electrical potential, the competition between these two elements leads to a local minimum in sedimentation potential as $\kappa a$ varies. For fixed surface potential, the sedimentation potential increases with $\kappa a$ due to the current arising from the diffusive element increasing with $\kappa a$. The local maximum of $\left|E^{*} / U^{*}\right|$ in Fig. 5 can be explained by the dependence of the sedimentation potential on both the surface potential and the thickness of the double layer.

For comparison, the values of $U / U_{0}$ and $E^{*} / U^{*}$ under the Debye-Hückel condition, that is, the nonlinear function of $\phi_{1}^{*}$ in Eq. [7] is linearized, are also presented in Figs. 3 and 5. These figures suggest that using the linearized Poisson-Boltzmann equation is inappropriate if $A$ is large.

The variation of scaled surface potential $\phi_{1 \mathrm{~S}}^{*}$ as a function of $\kappa a$ at various $B\left(=\left[\mathrm{H}^{+}\right]_{0} / K_{a}\right)$ is shown in Fig. 6. As can be seen from this figure, for a fixed $\kappa a$ the smaller the value of $B$, the higher the value of $\left|\phi_{1 \mathrm{~S}}^{*}\right|$. This is because the larger the value of $B$, the higher the bulk concentration of $\mathrm{H}^{+}$(or the lower the $\mathrm{pH}$ ), which yields a higher surface concentration of $\mathrm{H}^{+}$as suggested by Eq. [A2], and a lower [ $\left.\mathrm{A}^{-}\right]$according to Eq. [1], and, therefore, a lower surface charge density.

\section{Effect of $B$}

Figure 7 shows the variation of the scaled sedimentation velocity, $U / U_{0}$, as a function of $\kappa a$ at various $B$. This figure reveals that $U / U_{0}$ has a local minimum and a local maximum as $\kappa a$ varies. This can be explained by a reasoning similar to that presented in the discussion of Fig. 3.

The variation of the scaled sedimentation potential, $E^{*} / U^{*}$, as a function of $\kappa a$ at various $B$ is illustrated in Fig. 8. This figures reveals that if $B$ is small, $E^{*} / U^{*}$ has a local minimum as $\kappa a$ varies. However, if $B$ is large, $E^{*} / U^{*}$ increases monotonically with $\kappa a$. This can be explained by a reasoning similar to that presented in the discussion of Fig. 5.

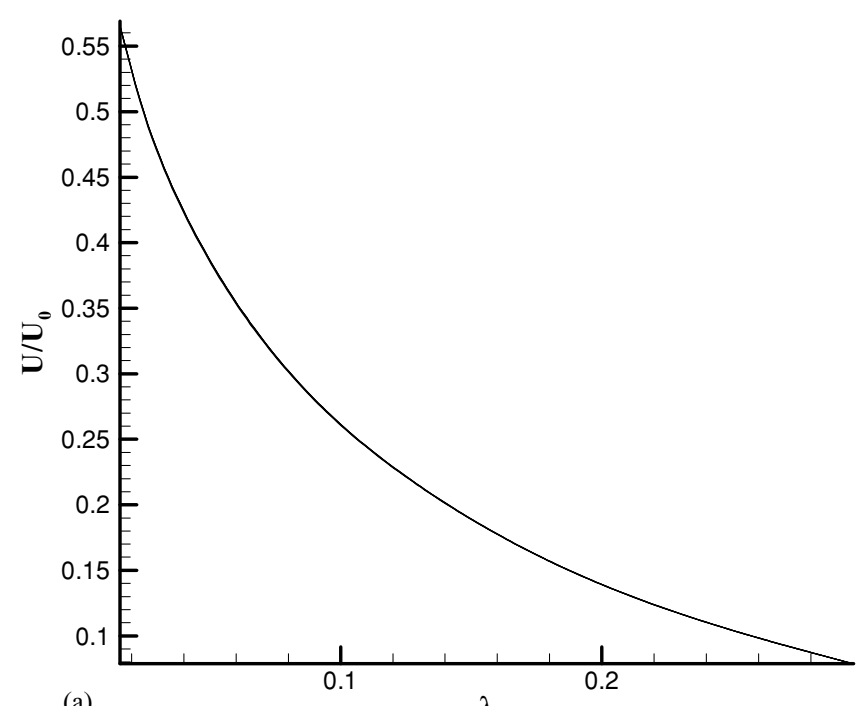

(a)

$\lambda$

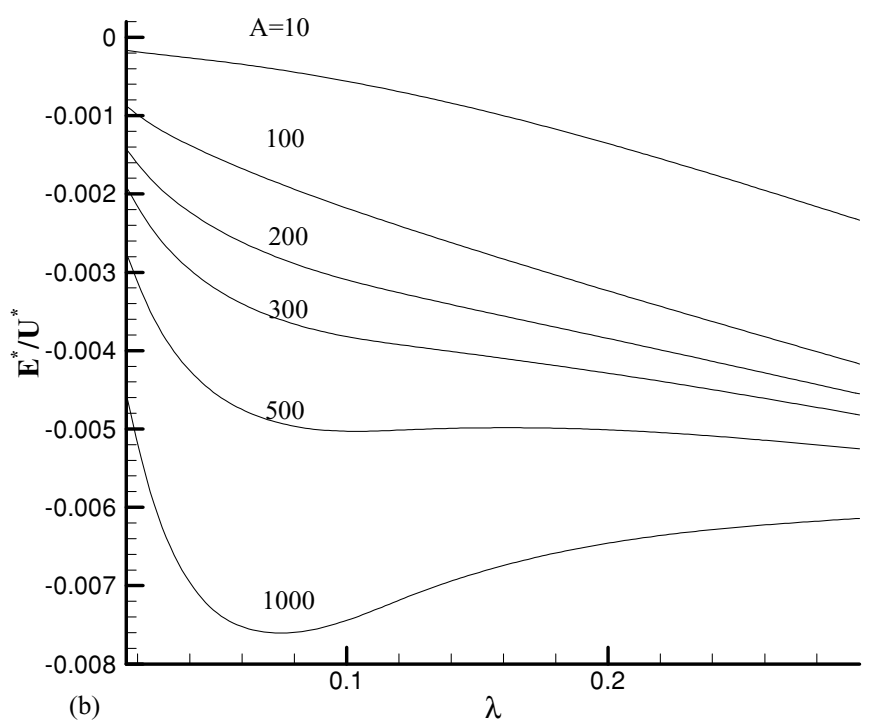

FIG. 9. Variation of scaled sedimentation velocity $U / U_{0}$ (a) and scaled sedimentation potential $E^{*} / U^{*}$ (b) as a function of $\lambda$ at various $A$ for the case $\kappa a=1.0, B=10$, and $\mathrm{Pe}_{1}=\mathrm{Pe}_{2}=0.01$.

\section{Effect of Volume Fraction}

Figure 9 shows the variation of the scaled sedimentation velocity $U / U_{0}$ and the scaled sedimentation potential $E^{*} / U^{*}$ as a function of the concentration of particle $\lambda$ at various $A$ for the case of a smaller $\kappa a$, and that for a larger $\kappa a$ is illustrated in Fig. 10a. Figures $9 \mathrm{a}$ and $10 \mathrm{a}$ reveal that $U / U_{0}$ decreases with the increase in $\lambda$. This is mainly due to the fact that the larger the concentration of particles, the more serious the interaction between neighboring particles. Figures $9 \mathrm{a}$ and 10a also indicate that the effect of $\kappa a$ on $U / U_{0}$ is much less significant than that of $\lambda$. As can be seen from Fig. 10b, $\left|E^{*} / U^{*}\right|$ increases with the increase in $\lambda$. According to its definition, the scaled sedimentation potential can be expressed as the ratio $\langle i\rangle_{1} /\langle i\rangle_{2}$, $\langle i\rangle_{1}$ and $\langle i\rangle_{2}$ being the net currents across the plane $\theta=\pi / 2$ in 
problems 1 and 2, respectively. The former is proportional to the sedimentation velocity, and the latter is proportional to the induced electric strength. The net current comprises that contributed by the ions inside the double layer and that contributed by the ions outside the double layer. If $\kappa a$ is large, the latter dominates. As $\lambda$ increases, since the amount of electrolyte outside the double layer decreases, the absolute current decreases accordingly. Also, this effect is more significant in problem 2 than that in problem 1 , and therefore, $\left|E^{*} / U^{*}\right|$ increases with the increase in $\lambda$. In Fig. $9 \mathrm{~b}, \kappa a=1$, which implies that the double layer is much thicker than that in Fig. 10b. In this case, the contribution of ions inside double layer to net current becomes more significant than that in Fig. 10b. If $A$ is small, the result shown in Fig. 9b is similar to that shown in Fig. 10b. However, if $A$ is large, $\left|E^{*} / U^{*}\right|$ has a local maximum as $\lambda$ varies. This can
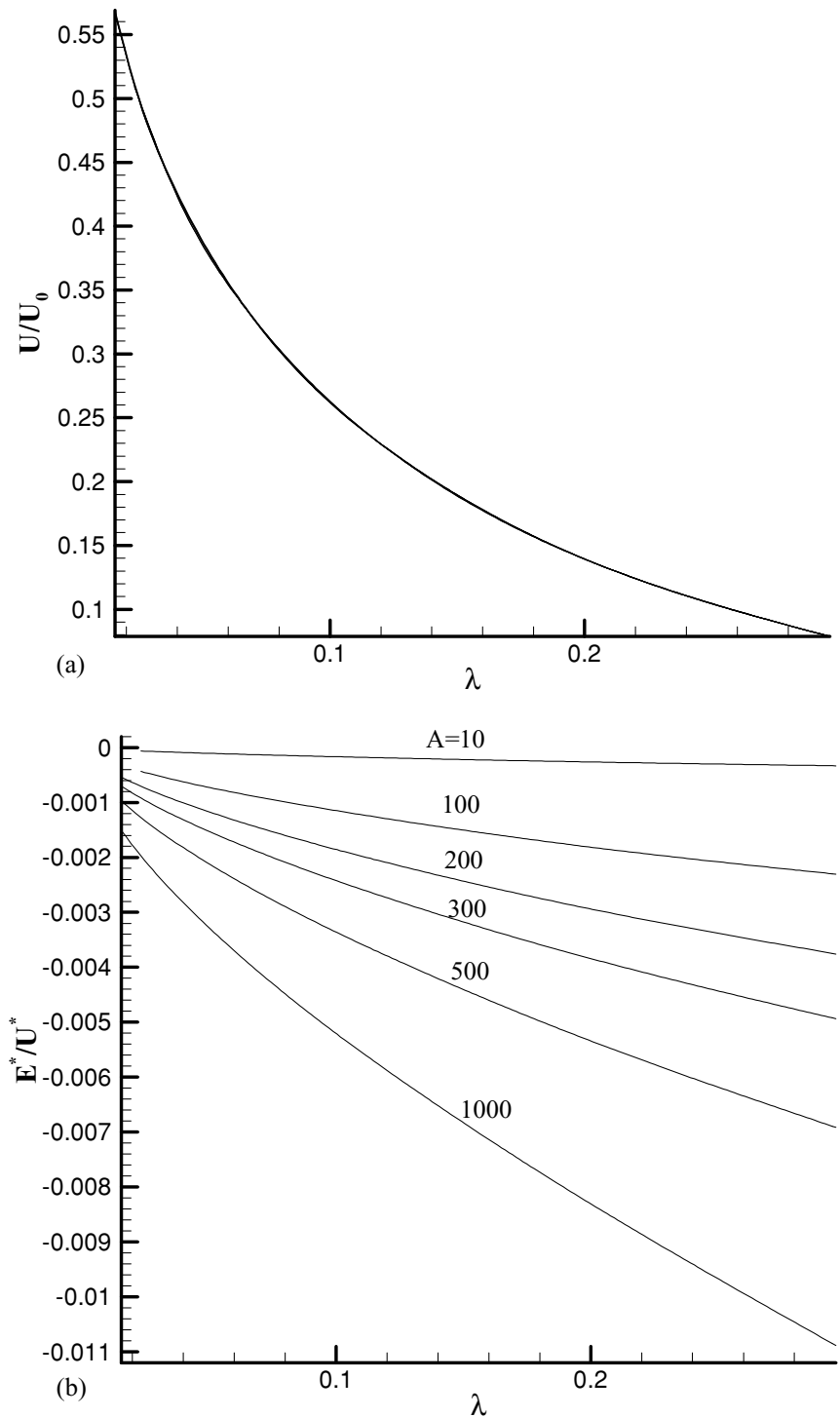

FIG. 10. Variation of scaled sedimentation velocity $U / U_{0}$ (a) and scaled sedimentation potential $E^{*} / U^{*}$ (b) as a function of $\lambda$ at various $A$ for the case $\kappa a=10, B=10$, and $\mathrm{Pe}_{1}=\mathrm{Pe}_{2}=0.01$.

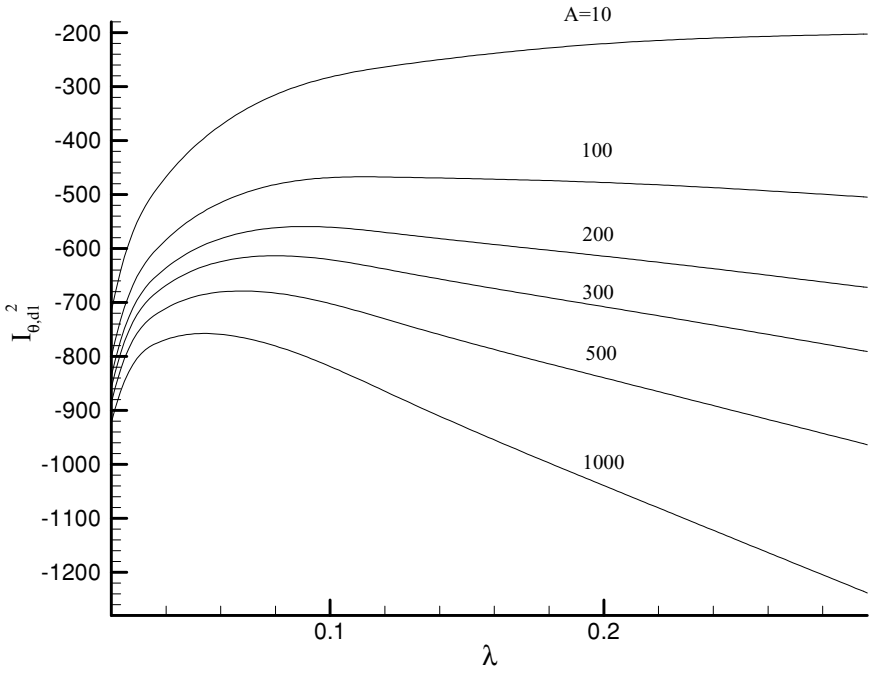

FIG. 11. Variation of diffusion current of cations of problem $2, I_{\mathrm{d} 1}^{2}$, as a function of $\lambda$ at various $A$ for the case $\kappa a=1.0, B=10$, and $\mathrm{Pe}_{1}=\mathrm{Pe}_{2}=0.01$.

be explained as follows. As pointed above, the amount of electrolyte outside the double layer decreases with the increase in $\lambda$, and the absolute current decreases. However, as $\lambda$ increases, the overlapping between adjacent double layers is significant, and the contribution to the current by the electrolyte outside the double layer becomes unimportant. The current inside the double layer is determined mainly by the surface potential of a particle. As shown in Fig. 2, the smaller the value of $A$, the lower the absolute surface potential, and therefore, the less significant its effect on the current. On the other hand, if $A$ is large, the absolute surface potential becomes high. In this case, the larger the $\lambda$, the greater the gradient of electrical potential, which leads to a larger absolute current contributed by counterions, as illustrated in Fig. 11, where

$$
I_{\theta, \mathrm{d} 1}^{2}=-\mathrm{Pe}_{1} \exp \left(-\phi_{1}^{*}\right) G_{1}
$$

is the current arising from the diffusive motion of cations in problem 2. Again, this effect for the case of problem 2 is more significant than that for the case of problem 1 .

\section{CONCLUSION}

The sedimentation of concentrated, charge-regulated spherical particles is analyzed theoretically. We show that, due to the complicated behaviors of the electric and hydrodynamic forces exerted on a particle, the sedimentation velocity of a particle exhibits both a local minimum and a local maximum as the thickness of the double layer $\kappa a$ varies. The sedimentation velocity approaches a constant value for both $\kappa a \rightarrow 0$ and $\kappa a \rightarrow \infty$; the limiting values are independent of the concentration of the dissociable functional groups on the particle surface, $N_{\mathrm{s}}$. As $\kappa a \rightarrow 0$, the sedimentation potential approaches a constant value, which is also independent of $N_{\mathrm{s}}$. If $N_{\mathrm{s}}$ is small, the 
absolute sedimentation potential decreases monotonically with $\kappa a$. However, if $N_{\mathrm{s}}$ is large, it may have a local maximum as $\kappa a$ varies. If $\mathrm{pH}$ is high, the sedimentation potential has a local minimum as $\kappa a$ varies. However, if $\mathrm{pH}$ is low, it increases monotonically with $\kappa a$. The sedimentation velocity decreases with the concentration of particle $\lambda$. If $N_{\mathrm{s}}$ is small, the absolute sedimentation potential increases with the increase in $\lambda$, but if $N_{\mathrm{s}}$ is large, it exhibits a local maximum as $\lambda$ varies.

\section{APPENDIX}

The equilibrium constant of the dissociation reaction expressed in Eq. [1] can be expressed as

$$
K_{a}=\frac{\left[\mathrm{A}^{-}\right]\left[\mathrm{H}^{+}\right]_{\mathrm{s}}}{[\mathrm{AH}]},
$$

where a symbol enclosed in square brackets represents the concentration, $\left[\mathrm{H}^{+}\right]_{\mathrm{S}}$ is the concentration of $\mathrm{H}^{+}$at the particle surface. We assume that the spatial distribution of $\left[\mathrm{H}^{+}\right]$can be described by the Boltzmann distribution

$$
\left[\mathrm{H}^{+}\right]_{\mathrm{s}}=\left[\mathrm{H}^{+}\right]_{0} \exp \left(-e \phi_{1} / k_{\mathrm{B}} T\right),
$$

where $\left[\mathrm{H}^{+}\right]_{0}$ is the equilibrium value of $\left[\mathrm{H}^{+}\right]$. The concentration of the functional groups on particle surface, $N_{\mathrm{s}}$, can be evaluated by

$$
N_{\mathrm{s}}=\left[\mathrm{A}^{-}\right]+[\mathrm{AH}] .
$$

Equations [A1]-[A3] lead to

$$
\left[\mathrm{A}^{-}\right]=\frac{N_{\mathrm{s}}}{1+\left(\left[\mathrm{H}^{+}\right]_{0} / K_{a}\right) \exp \left(-e \phi_{1} / k_{\mathrm{B}} T\right)} .
$$

The charge density on particle surface, $\sigma$, can be expressed by

$$
\sigma=-e\left[\mathrm{~A}^{-}\right]
$$

Substituting Eq. [A3] into Eq. [A4], we obtain

$$
\sigma=-\frac{e N_{\mathrm{s}}}{1+\left(\left[\mathrm{H}^{+}\right]_{0} / K_{a}\right) \exp \left(-e \phi_{1} / k_{\mathrm{B}} T\right)} .
$$

Suppose that the relative permittivity of the liquid phase is much larger than that of the dispersed phase. Then

$$
\sigma=-\varepsilon\left(\frac{d \phi_{1}}{d r}\right)_{r=a} .
$$

Equations [A6] and [A7] yield

$$
\begin{aligned}
\frac{d \phi_{1}^{*}}{d r^{*}} & =\frac{e^{2} N_{\mathrm{s}} a / \varepsilon k_{\mathrm{B}} T}{1+\left(\left[\mathrm{H}^{+}\right]_{0} / K_{a}\right) \exp \left(-\phi_{1}^{*}\right)} \\
& =\frac{A}{1+B \exp \left(-\phi_{1}^{*}\right)}, \quad r^{*}=1,
\end{aligned}
$$

where $r^{*}=r / a, \quad \phi_{1}^{*}=\phi_{1} /\left(k_{\mathrm{B}} T / e\right), \quad A=e^{2} N_{\mathrm{s}} a / \varepsilon k_{\mathrm{B}} T$, and $B=\left[\mathrm{H}^{+}\right]_{0} / K_{a}$. Note that if $K_{a} \rightarrow \infty, B \rightarrow 0$, and Eq. [A8] becomes

$$
\frac{d \phi_{1}^{*}}{d r^{*}}=A, \quad r^{*}=1
$$

which corresponds to a constant charge boundary condition. On the other hand, if $K_{a} \rightarrow 0, B \rightarrow \infty$, and Eq. [A8] reduces to

$$
\frac{d \phi_{1}^{*}}{d r^{*}}=0, \quad r^{*}=1
$$

which implies that $\phi_{1}^{*}$ is constant.

We introduce the stream function representation to eliminate the pressure term in Eq. [5], and the result is

$$
\mathbf{i}_{\phi} E^{4} \psi=-\frac{1}{\eta} \sin \theta \nabla \times\left(\rho \nabla\left(\phi_{1}+\phi_{2}\right)\right),
$$

where $\psi$ is the stream function, $\mathbf{i}_{\phi}$ is the unit vector in the $\phi$ direction, $E^{4}=E^{2} E^{2}$ with

$$
E^{2}=\frac{\partial^{2}}{\partial r^{2}}+\frac{\sin \theta}{r^{2}} \frac{\partial}{\partial \theta}\left(\frac{1}{\sin \theta} \frac{\partial}{\partial \theta}\right) .
$$

In the discussion below, scaled variables, which are represented by a symbol with an asterisk, are used. The applied electric field is assumed to be weak so that the relevant governing equations can be linearized. Suppose that the scaled equilibrium potential, $\phi_{1}^{*}$, can be described by the scaled form of Eq. [3] with the scaled number concentrations of ions $n_{1}^{*}=\exp \left(-\phi_{1}^{*}\right)$ and $n_{2}^{*}=\exp \left(\alpha \phi_{1}^{*}\right)$ as

$$
\nabla^{* 2} \phi_{1}^{*}=-\frac{(\kappa a)^{2}}{(1+\alpha)}\left[\exp \left(-\phi_{1}^{*}\right)-\exp \left(\alpha \phi_{1}^{*}\right)\right] .
$$

Since $\nabla^{* 2} \phi_{2}=\nabla^{* 2} \phi-\nabla^{* 2} \phi_{1}$, applying Eqs. [3], [7], and Eq. [A12] gives

$$
\begin{gathered}
\nabla^{* 2} \phi_{2}^{*}-\frac{(\kappa a)^{2}}{(1+\alpha)}\left[\exp \left(-\phi_{1}^{*}\right)+\alpha \exp \left(\alpha \phi_{1}^{*}\right)\right] \phi_{2}^{*} \\
=\frac{(\kappa a)^{2}}{(1+\alpha)}\left[\exp \left(-\phi_{1}^{*}\right) g_{1}^{*}+\exp \left(\alpha \phi_{1}^{*}\right) \alpha g_{2}^{*}\right]
\end{gathered}
$$

Substituting Eq. [7] into Eq. [2] and neglecting the terms that involve the product of two perturbed terms yields

$$
\begin{gathered}
\nabla^{* 2} g_{1}^{*}-\nabla^{*} \phi_{1}^{*} \bullet \nabla^{*} g_{1}^{*}=\operatorname{Pe}_{1} \mathbf{v}^{*} \bullet \nabla^{*} \phi_{1}^{*} \\
\nabla^{* 2} g_{2}^{*}+\alpha \nabla^{*} \phi_{1}^{*} \bullet \nabla^{*} g_{2}^{*}=\operatorname{Pe}_{2} \mathbf{v}^{*} \bullet \nabla^{*} \phi_{1}^{*} .
\end{gathered}
$$


Substituting Eq. [7] into Eq. [A11] gives

$$
E^{* 4} \psi^{*}=\frac{(\kappa a)^{2}}{(1+\alpha)}\left[\left(\frac{\partial g_{1}^{*}}{\partial \theta} n_{1}^{*}+\frac{\partial g_{2}^{*}}{\partial \theta} \alpha n_{2}^{*}\right) \frac{\partial \phi_{1}^{*}}{\partial r^{*}}\right] \sin \theta .
$$

Equations [9]-[12] can be recovered by applying the relations $\phi_{2}^{*}=\Phi_{2}(r) \cos \theta, \quad g_{1}^{*}=G_{1}(r) \cos \theta, \quad g_{2}^{*}=G_{2}(r) \cos \theta, \quad$ and $\psi^{*}=\Psi(r) \sin ^{2} \theta$ to Eqs. [A13]-[A17].

\section{ACKNOWLEDGMENT}

This work is supported by the National Science Council of the Republic of China.

\section{REFERENCES}

1. von Smoluchowski, M., Z. Phys. Chem. 92, 129 (1918).

2. Booth, F., J. Chem. Phys. 22, 1956 (1954).

3. Booth, F., Proc. R. Soc. London Ser. A 203, 514 (1950).

4. Overbeek, J. Th. G. Kolloid-Beih. 54, 287 (1943).

5. Saville, D. A., Adv. Colloid Interface Sci. 16, 267 (1982).
6. Ohshima, H., Healy, T. W., White, L. R., and O'Brien, R. W., J. Chem. Soc., Faraday Trans. 2 80, 1299 (1984).

7. Keh, H. J., and Ding, J. M., J. Colloid Interface Sci. 227, 540 (2001).

8. Pujar, N. S., and Zydney, A. L., Am. Inst. Chem. Engrg. J. 42, 2101 (1996).

9. Ninham, B. W., and Parsegian, V. A., J. Theor. Biol. 31, 405 (1971).

10. Krozel, J. W., and Saville, D. A., J. Colloid Interface Sci. 150, 365 (1992).

11. Carnie, S. L., and Chan, D. Y. C., J. Colloid Interface Sci. 155, 297 (1993).

12. Carnie, S. L., Chan, D. Y. C., and Gunning, J. S., Langmuir 10, 2993 (1994).

13. Carnie, S. L., Chan, D. Y. C., and Stankovich, J., J. Colloid Interface Sci. 165, 116 (1994).

14. Carnie, S. L., and Chan, D. Y. C., J. Colloid Interface Sci. 161, 260 (1993).

15. Prieve, D. C., and Ruckenstein, E., J. Theor. Biol. 56, 205 (1976).

16. Krozel, J. W., J. Colloid Interface Sci. 163, 437 (1994).

17. Ding, J. M., and Keh, H. J., J. Colloid Interface Sci. 243, 331 (2001).

18. Hunter, R. J., "Foundations of Colloid Science," Vol.1, Oxford Univ. Press, London, 1989.

19. Kuwabara, S., J. Phys. Soc. Jpn. 14, 527 (1959).

20. O'Brien, R. W., and White, L. R., J. Chem. Soc. Faraday Trans. 2 74, 1607 (1978).

21. Lee, E., Chu, J. W., and Hsu, J. P., J. Chem. Phys. 110, 11,643 (1999).

22. Levine, S., and Neale, G. H., J. Colloid Interface Sci. 47, 520 (1974).

23. Hsu, J. P., Lee, E., and Yen, F. Y., J. Chem. Phys. 112, 6404 (2000). 